\title{
Discrete-Spectrum Waves in the Vicinity of Cyclotron Resonance in Silver
}

\author{
Henningsen, J. 0.
}

Published in:

Physical Review Letters

Link to article, DOI:

10.1103/PhysRevLett.24.823

Publication date:

1970

Document Version

Publisher's PDF, also known as Version of record

Link back to DTU Orbit

Citation $(A P A)$ :

Henningsen, J. O. (1970). Discrete-Spectrum Waves in the Vicinity of Cyclotron Resonance in Silver. Physical Review Letters, 24(15), 823-826. https://doi.org/10.1103/PhysRevLett.24.823

\section{General rights}

Copyright and moral rights for the publications made accessible in the public portal are retained by the authors and/or other copyright owners and it is a condition of accessing publications that users recognise and abide by the legal requirements associated with these rights.

- Users may download and print one copy of any publication from the public portal for the purpose of private study or research.

- You may not further distribute the material or use it for any profit-making activity or commercial gain

- You may freely distribute the URL identifying the publication in the public portal

If you believe that this document breaches copyright please contact us providing details, and we will remove access to the work immediately and investigate your claim. 


\title{
DISCRETE-SPECTRUM WAVES IN THE VICINITY OF CYCLOTRON RESONANCE IN SILVER
}

\author{
J. O. Henningsen
}

Physics Laboratory I, Technical University of Denmark, DK-2800 Lyngby, Denmark

(Received 11 November 1969)

\begin{abstract}
Cyclotron-resonance studies of silver have revealed two additional series of oscillations, one between the fundamental and the second harmonic, the other above the fundamental resonance. These series are caused by the excitation of weakly damped discretespectrum waves propagating perpendicular to the static magnetic field.
\end{abstract}

Recording the [100] cyclotron-resonance spectrum of silver, we observed two additional sets of oscillations, one between the fundamental and the second harmonic, and the other above the fundamental resonance. The cyclotron-resonance signals are due to belly orbits, and are adequately described by the Azbel'-Kaner theory. ${ }^{1}$ We show that the additional sets are caused by weakly damped electromagnetic excitations called discrete-spectrum waves. ${ }^{2,3}$

The experimental results are shown in Fig. 1. This is not the first observation of cyclotron resonance in silver, but previous measurements ${ }^{4}$ did not cover the range of magnetic field above the second harmonic in which we see the additional oscillations. Our experiments were performed at 45.2 and $70.2 \mathrm{GHz}$. In both cases we used superheterodyne reflection spectrometers and 270$\mathrm{Hz}$ magnetic field modulation. The temperature was $4.2^{\circ} \mathrm{K}$. The sample surface was parallel to a (110) plane, the static magnetic field pointed in the [100] direction, and the rf electric field was polarized in the [110] direction. The additional signals were strongest when the magnetic field was parallel to the surface. A tilt of $i^{\circ}$ led to a shift of $2 \%$ towards lower magnetic fields, and at the same time it reduced the amplitudes considerably. Two different samples of thickness 0.7 and $0.45 \mathrm{~mm}$ gave identical results. Both of them had a resistivity ratio of 7500 . No special precautions were taken to make them plane parallel, and they were only polished on one side.

The concept of discrete-spectrum waves was introduced for a free-electron model by Kaner and Skobov. ${ }^{2}$ They noted that a wave can propagate weakly damped in a direction almost perpendicular to a static magnetic field if an integral number of wavelengths fit into the orbit diameter of electrons from the central part of the Fermi surface. ${ }^{5}$ This geometric condition selects a discrete set of allowed wavelengths. The physical

\section{Discrete Spectrum Waves}

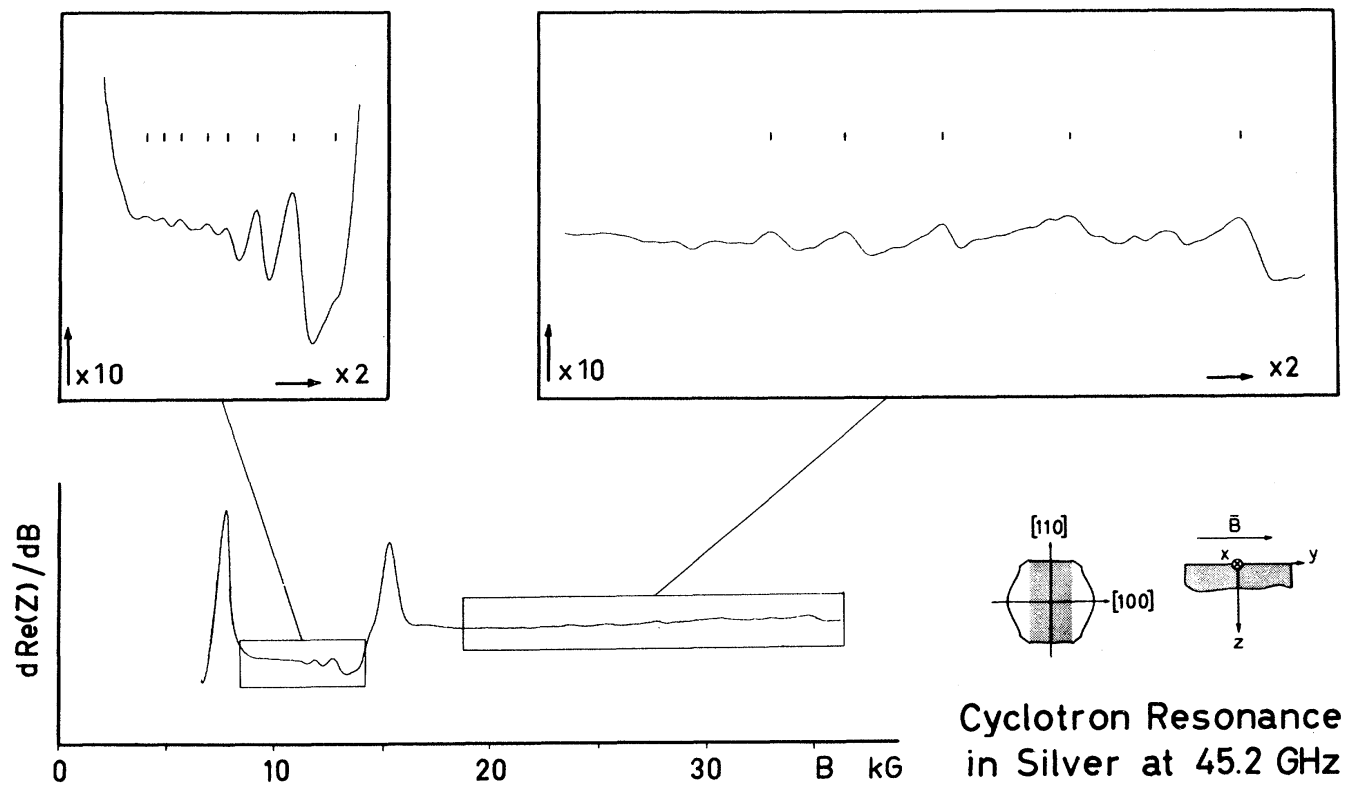

FIG. 1. Recording of discrete-spectrum waves in silver. One series is seen between the fundamental and the second harmonic of cyclotron resonance, the other above the fundamental. 
origin of the nondamping condition is that the interaction between electron and wave cancels out at the two turning points of the orbit. Here the interaction is particularly strong because the electron moves in a plane of stationary phase. In a magnetic field parallel to the surface all electrons contribute to the effective conductivity. However, their orbit diameters vary over the Fermi surface, and the nondamping condition can not be simultaneously met by them all. By tilting the magnetic field slightly, the contribution from the noncentral electrons is reduced because of their drift velocity. The effective conductivity is now dominated by the central electrons which all have nearly the same orbit diameter, and wave propagation is possible. The original work of Kaner and Skobov dealt with the low-frequency case $\omega \ll \omega_{c}$. Later Blank and Kaner ${ }^{3}$ showed that similar waves exist in the vicinity of each of the cyclotron-resonance harmonics $\omega \simeq n \omega_{c}$, and this prediction has recently been experimentally confirmed. ${ }^{6}$

It follows that discrete-spectrum waves can propagate if a group of electrons with nearly the same orbit diameters dominate the effective conductivity. In the high-frequency case they must have the same cyclotron frequency as well. The electrons between the necks of the silver Fermi surface (the shaded area in Fig. 1) constitute, to a good approximation, such a group. ${ }^{7}$ By using a simplified Fermi-surface model in which the remaining electrons are neglected, we show that discrete-spectrum waves can propagate even when the magnetic field is parallel to the surface. Further, we show that this causes a periodic structure in the surface impedance, and we compare the period of this structure with our experimental results.

Let us begin by considering wave propagation in the infinite metal. The wave numbers of the propagating modes must satisfy the dispersion equation

$$
q^{2}-i \omega \mu \sigma(q)=0
$$

where $\sigma(q)$ is the Fourier transform of the nonlocal conductivity appropriate to the infinite medium. The space and time dependence of the wave, propagating along the $z$ axis, is given by $\exp [i(q z-\omega t)]$. The particular features of the silver Fermi surface enter in the calculation of the conductivity. Treating the section between the necks as a cylinder and neglecting the remaining part, we obtain, in the asymptotic limit $(q D)^{1 / 2}$ $\gg 1$ (mks units),

$$
\begin{aligned}
& \sigma_{x x}(q)=\frac{s^{3}}{\omega \mu}\left[\frac{1}{|q|} \operatorname{coth}(\pi \gamma)-\frac{1}{q} \frac{\sin (q D)}{\sinh (\pi \gamma)}\right], \\
& \sigma_{x y}=\sigma_{y x}=\sigma_{y y}=0, \\
& \gamma=-i \omega / \omega_{c}+1 / \omega_{c} \tau
\end{aligned}
$$

(compare with Chamber $\mathrm{s}^{8}$ ). The $x$ axis is perpendicular to the direction of propagation and to the magnetic field. The constant $s$ is proportional to the reciprocal skin depth and is essentially determined by the Fermi-surface parameters.

For fixed $q$, the conductivity oscillates with two periods in $1 / B$. The larger of these comes from the factors $\operatorname{coth}(\pi \gamma)$ and $1 / \sinh (\pi \gamma)$, and reflects the temporal resonance between the cyclotron frequency and the frequency of the electromagnetic field. The smaller period comes from the factor $\sin (q D)$, and reflects the spatial resonance between the wavelength of the Fourier component and the orbit diameter of the electrons. For a free-electron model, the integration over different orbit diameters will introduce an additional factor of $(q D)^{-1 / 2}$ in front of $\sin (q D)$. In the limit $(q D)^{1 / 2} \gg 1$, this will destroy the effect of the spatial resonance as long as the magnetic field is kept perpendicular to the direction of propagation.

We discuss the solutions to the dispersion equation in the limit of an infinite relaxation time. $\sigma(q)$ is then purely imaginary. Figure 2 shows, for a fixed magnetic field, the functions $q^{2}$ and $i \omega \mu \sigma(q)$ for real, positive $q$. Each of the intersections corresponds to an undamped propagating wave. Assuming the surface scattering to be specular, we can estimate the influence of such waves on the surface impedance of a semi-infinite metal. Under this assumption, the expression (2) for the conductivity holds for all $z>0$,

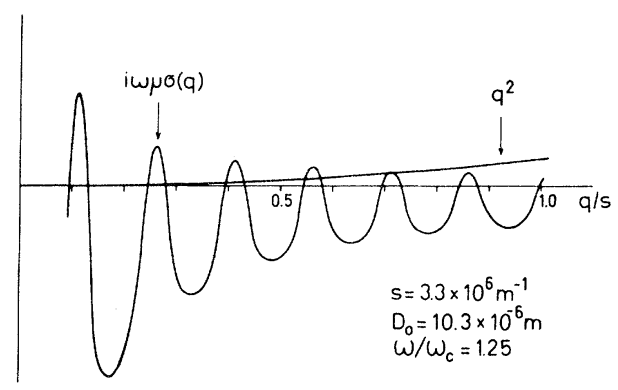

FIG. 2. Graphical solution of the infinite-medium dispersion equation for fixed $\omega$ and $B$ in the limit $\omega \tau$ $\rightarrow \infty$. Intersections correspond to undamped modes. Surface-impedance anomalies occur when the two curves touch. 
and the surface impedance is given $b y^{8}$

$$
Z=-\frac{2 i \omega \mu}{\pi} \int_{0}^{\infty} \frac{d q}{q^{2}-i \omega \mu \sigma(q)}
$$

Here the solutions to the infinite-medium dispersion equation appear as poles of the integrand. A change in magnetic field causes a change in the period and the amplitude of $\sigma(q)$. This is accompanied by a smooth change in the surface impedance until one of the peaks of $i \omega \mu \sigma(q)$ just touches $q^{2}$. When this condition is fulfilled, the dispersion equation is satisfied for a finite range of $q$ values, and we get a sudden increase in $Z$. This is known from helicon propagation in a semiinfinite metal, where the touching of the two functions gives rise to the so-called edge anomaly in the surface impedance. ${ }^{9,10}$ In terms of the dispersion law of the waves, the condition corresponds to $d \omega / d q=0$. This means that an undamped wave causes a change in the surface impedance at the moment when its group velocity becomes zero. In the present case the condition is periodically fulfilled because of the oscillatory character of $\sigma(q)$. To a good approximation the touching points correspond to $\sin (q D)= \pm 1$, the upper sign valid for $2 n<\omega / \omega_{c}<2 n+1$, the lower for $2 n+1<\omega / \omega_{c}<2 n+2$, where $n$ is an integer.

Inserting this in the dispersion equation (1) we get, for $\omega \tau \rightarrow \infty,{ }^{11}$

$$
\begin{aligned}
& 2 \pi\left(p-\frac{3}{4}\right)=s D_{0} \xi\left(\frac{\cos \pi \xi-1}{-\sin \pi \xi}\right)^{1 / 3}, 2 n<\xi<2 n+1 \\
& 2 \pi\left(p-\frac{1}{4}\right)=s D_{0} \xi\left(\frac{\cos \pi \xi+1}{-\sin \pi \xi}\right)^{1 / 3}, 2 n+1<\xi<2 n+2 \\
& n=0,1,2, \cdots, \\
& p=3,4,5, \cdots,
\end{aligned}
$$

where $\xi=\omega / \omega_{c}$ and $D_{0}$ is the orbit diameter of the electrons at the fundamental cyclotron resonance. By solving for $\xi$ we determine the position of the $p$ th surface-impedance anomaly in units of $\omega / \omega_{c}$.

We are now able to relate the experimental observations to our theoretical model. In Fig. 3, the right-hand side of (4), taken at the observed peak positions, is plotted versus an integer $p$. The points lie on reasonably straight lines, although II shows some deviation for low $\xi$. $p$ denotes the number of wavelengths enclosed by an electron orbit. At $45.2 \mathrm{GHz} D_{0}$ is approximately $10 \mu$, and a typical wavelength of the discretespectrum waves is therefore of the order of $1 \mu$. From the slope of the lines we can determine the parameter $s$. Using $k_{\mathrm{F}}=1.20 \times 10^{10} \mathrm{~m}^{-1},{ }^{6}$ we arrive at the values indicated in the figure. If we take the width of the cylindrical Fermi surface section as $0.7 k_{\mathrm{F}}$, we get from a first-principles calculation at $45.2 \mathrm{GHz}$

$$
s=\left(\frac{1.4}{\pi}\right)^{1 / 3}\left(\frac{e^{2} k_{\mathrm{F}}^{2} \omega \mu}{4 \pi \hbar}\right)^{1 / 3}=7.6 \times 10^{6} \mathrm{~m}^{-1}
$$

$s_{\text {I }}$ agrees very well with this result, whereas $s_{\text {II }}$ is too small. From (5) we see that $s$ is proportional to $\omega^{1 / 3}$. We thus expect a ratio of $s_{\text {III }} / s_{\text {II }}$ $=1.16$, which is slightly smaller than the observed ratio of 1.24. We believe that the discrepancies are mainly caused by the oversimplified Fermi-surface model. In our calculation of the conductivity we neglected the electrons from outside the cylindrical section. These electrons do not give rise to resonances because they cover a wide range of orbit diameters and cyclotron frequencies. However, they will add a slowly varying contribution to the conductivity. A quantitative estimate indicates that the inclusion of such a contribution in (4) will leave $s_{\text {I }}$ essentially un-

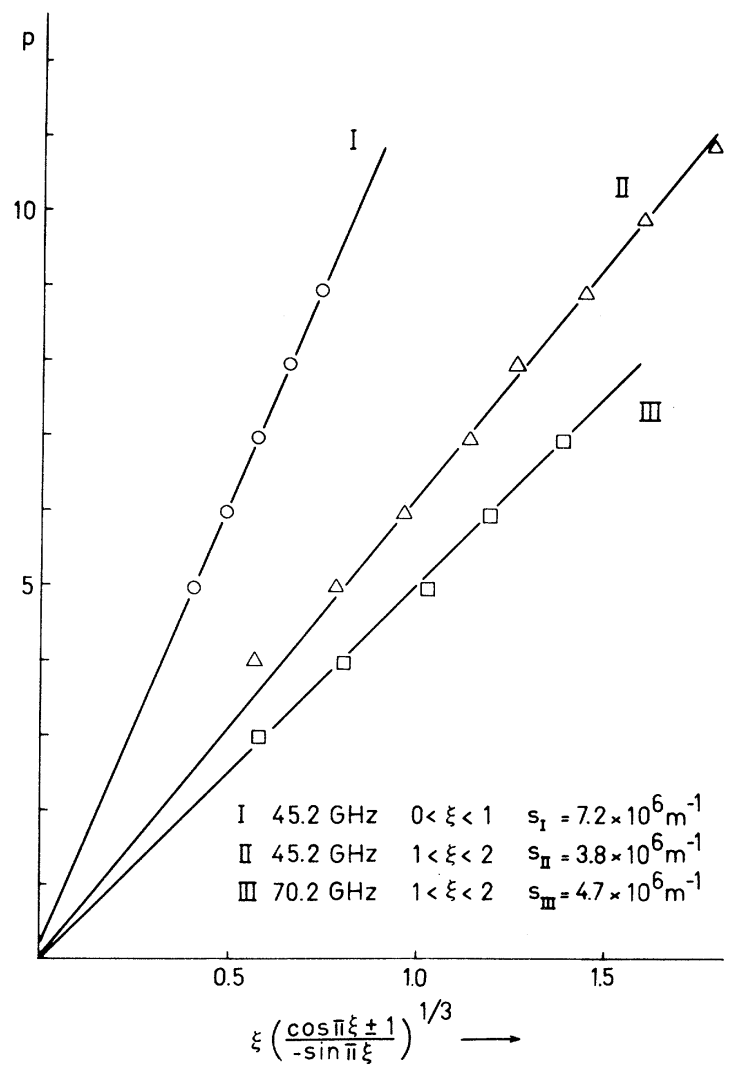

FIG. 3. Observed values of $\xi[(\cos \pi \xi \pm 1) /(-\sin \pi \xi)]^{1 / 3}$ plotted versus an integer $p . \xi_{p}$ is the position of the $p$ th surface impedance anomaly expressed in units of $\omega / \omega_{c}$. The upper sign is used for $1<\omega / \omega_{c}<2$, the lower for $0<\omega / \omega_{c}<1$. 
changed, whereas $s_{\text {II }}$ will be shifted upwards by a substantial amount.

It may be instructive to compare the present case with experiments on plane-parallel slabs which are normally used in investigation of wave propagation. In the general case the wave number and damping of the propagating waves vary smoothly with the magnetic field, and no structure will be seen in the surface impedance unless a standing wave is set up. In a slab experiment this is done by reflection from the second boundary, and the allowed wavelengths are determined by the slab thickness. In our case the standing wave is set up in the bulk metal by the simultaneous excitation of two waves of the same frequency but opposite wave number, each of them having zero group velocity. ${ }^{9}$ The observed surface-impedance anomalies therefore correspond to standing-wave resonances of the infinite metal, and the allowed wavelengths are determined by the orbit diameter of the electrons.

It follows from the preceding discussion that the particular shape of the silver Fermi surface allows discrete-spectrum waves to propagate in a magnetic field parallel to the sample surface. It follows further that the periods of the observed surface-impedance oscillations can be related to the infinite-medium dispersion equation. However, we cannot draw any conclusions regarding the amplitudes of the oscillations from the expression (3) for the surface impedance. This expression rests upon the assumption that all electrons interacting with the surface are scattered specularly, regardless of their impact angle. This is physically unrealistic. When we are less than an orbit diameter away from the surface, the diffusely scattered electrons introduce a cou- pling between normal modes of different wave numbers. This invalidates the concept of independent propagating modes. Moreover it gives a real contribution to the conductivity so that any wavelike disturbance will immediately be damped. The discrete-spectrum waves therefore only exist in the bulk region $z>D$. As a possible excitation mechanism, we suggest that the waves are launched by electrons which skim through the skin layer and transfer $\mathrm{rf}$ energy down to $z=D$.

Thanks are due to Professor Dr. K. Saermark whose suggestions and comments have continuously forced me to evaluate the arguments. Also, I wish to thank Professor Dr. J. F. Koch who drew my attention to the analogy between the present problem and that of helicon propagation.

\footnotetext{
${ }^{1}$ M. Ya. Azbel' and E. A. Kaner, Zh. Eksperim. i Teor. Fiz. 30, 811 (1956) [Soviet Phys. JETP $\underline{5}, 730$ (1956)].

${ }^{2}$ E. A. Kaner and V. G. Skobov, Physics $\underline{2}, 165$ (1966).

${ }^{3}$ A. Ya. Blank and E. A. Kaner, Phys. Status Solidi 22, 47 (1967).

${ }^{4}$ D. G. Howard, Phys. Rev. 140 , A1705 (1965).

${ }^{5}$ The exact condition involves a phase constant of $\frac{1}{4}$.

${ }^{6}$ V. P. Naberezhnykh and N. K. Dan'shin, Zh. Eksperim. i Teor. Fiz.- Pis'ma Redakt. 10, 22 (1969) [JETP Letters 10, 14 (1969)].

${ }^{7}$ N. E. Christensen, Phys. Status Solidi 31, 635 (1969).

${ }^{8}$ R. G. Chambers, in Physics of Metals, edited by J. M. Ziman (Cambridge University, Cambridge, England, 1969), Vol. I, Electrons.

${ }^{9}$ J. C. McGroddy, J. L. Stanford, and E. A. Stern, Phys. Rev. 141, 437 (1966).

${ }^{10}$ D. S. Falk, B. Gerson, and J. F. Carolan, Phys. Rev. (to be published).

${ }^{11}$ The asymptotic expansion of $\sigma(q)$ is not valid for the smallest $p$ values.
} 